\title{
Intensity Dependent Effects in RHIC
}

\author{
Jie Wei \\ Brookhaven National Laboratory, Upton, New York 11973, USA ${ }^{1}$
}

\begin{abstract}
The Relativistic Heavy Ion Collider (RHIC) is currently under commissioning after a seven-year construction cycle. Unlike conventional hadron colliders, this machine accelerates, stores, and collides heavy ion beams of various combinations of species. The dominant intensity dependent effects are intra-beam scattering at both injection and storage, and complications caused by crossing transition at a slow ramp rate. In this paper, we present theoretical formalisms that have been used for our study, and discuss mechanisms, impacts, and compensation methods including beam cooling and transition jump schemes. Effects of space charge, beam-beam, and ring impedances are also summarized.
\end{abstract}

\section{INTRODUCTION}

The primary motivation for colliding heavy ions at ultra-relativistic energies is the belief that it is possible to create macroscopic volumes of nuclear matter at such extreme conditions of temperature and energy density that a phase transition will occur from hadronic matter to a confined plasma of quarks and gluons. The main goal of the Relativistic Heavy Ion Collider (RHIC) $[1,2]$ is to provide head-on collisions at energies up to $100 \mathrm{GeV} / \mathrm{u}$ per beam for various species from proton to gold. Luminosity requirements for the heaviest ions are specified to be in the $10^{26-27}$ $\mathrm{cm}^{-2} \mathrm{~s}^{-1}$ range. The higher gold-gold total cross-section results in interaction rates comparable to proton-proton colliders although this luminosity is several orders of magnitude lower than those machines. Based on these general requirements, RHIC machine parameters were derived and are outlined in Table 1.

Comparing with conventional proton colliders like the Tevatron and the Large Hadron Collider where limitation on beam lifetime and luminosity performance is due to beam-beam effects, performance of RHIC is primarily limited by intrabeam Coulomb scattering, which becomes increasingly important for high charge state ions. The scattering cross-section is proportional to $Z^{4} / A^{2}$ for particles of charge state $Z$ and atomic number $A$. For fully stripped gold ions, intra-beam scattering causes transverse emittance growth, increasing the emittance from 10 to more than $40 \mathrm{~mm} \cdot \mathrm{mr}$ in 10 hours, even in the optimal scenario when the transverse

1) Work performed under the auspices of the US Department of Energy. 
TABLE 1. Major parameters for the Relativistic Heavy Ion Collider.

Kinetic Energy, Injection - Top gold proton

Circumference, $2 \pi R$

Number of bunches per ring

Number of crossing points

$\beta^{*}$, injection

$\beta^{*}$, low-beta insertion

Transition energy, $\gamma_{T}$

Betatron tunes horizontal vertical

10.8-100 $\mathrm{GeV} / \mathrm{u}$

28.3-250 GeV

$3833.845 \mathrm{~m}$

60

6

$10 \quad \mathrm{~m}$

$1 \mathrm{~m}$

22.89

28.19

29.18

Magnetic rigidity, $B_{0} \rho$ injection top energy

Dipole field at top energy

Quadrupole gradient at top energy

Accelerating RF system:

harmonic number, $h$ maximum voltage, $V$

Storage RF system: harmonic number, $h$ maximum voltage, $V$

$97.5 \quad \mathrm{~T} \cdot \mathrm{m}$

$839.5 \quad \mathrm{~T} \cdot \mathrm{m}$

$3.45 \quad \mathrm{~T}$

$71.2 \quad \mathrm{~T} / \mathrm{m}$

360

0.6

MV

2520

6

MV

Intensity per bunch, $N$ gold

$10^{9}$

proton

$10^{11}$

Transverse emittance (95\% normalized), $\epsilon_{N}$ gold

$10-40 \quad \mathrm{~mm} \cdot \mathrm{mr}$ proton

$20-27$ $\mathrm{mm} \cdot \mathrm{mr}$

Longitudinal bunch area $(95 \%), S$ gold

$0.3-1.2 \mathrm{eV} \cdot \mathrm{s} / \mathrm{u}$ proton

$0.3-1$ $\mathrm{eV} \cdot \mathrm{s}$

Average luminosity: gold

$10^{26}$ $\mathrm{cm}^{-2} \mathrm{~s}^{-1}$ proton

$10^{31} \quad \mathrm{~cm}^{-2} \mathrm{~s}^{-1}$


motion is fully coupled [3]. This growth results in a large beam dimension in the interaction region focusing quadrupole triplets. Consequently, the impact from the field errors of these superconducting magnets becomes significant, limiting the lowest achievable $\beta^{*}$ to about 1 meter at the interaction point and hence limiting the highest achievable luminosity. Longitudinally, intra-beam scattering causes bunch area growth, resulting in intensity loss of as much as $40 \%$ in 10 hours as particles escape the confining buckets of the radio-frequency (RF) system. In order to reduce beam loss and to improve luminosity lifetime, beam cooling has been investigated $[4,17]$.

RHIC is the first superconducting machine where the beams have to be accelerated across the transition energy. Comparing with normal-conducting magnets, the superconducting magnets require a slow ramp rate for beam acceleration. Both chromatic nonlinear effects and beam self-field effects are strong during transition crossing [5]. A "matched first order" transition jump scheme is designed [6] to effectively increase the crossing rate by a factor of 8 during the $60 \mathrm{~ms}$ time around transition [7]. With such a scheme, the longitudinal emittance growth can be limited to less than $20 \%$ at transition with minimum disruption to the transverse particle motion.

This paper summarizes the dominant intensity dependent effects in RHIC. In Section 2, we review the scaling laws of intra-beam scattering, and discuss the Fokker-Planck formalism that describes the evolution of beam distribution and beam lifetime. In Section 3, we review formalisms for the non-adiabatic regime of transition crossing, emphasizing self-field mismatching and microwave instability. Space charge and beam-beam effects are briefly discussed in Section 4. Impedances and more conventional instabilities [8] are discussed in Section 5. The conclusion is given in Section 6.

\section{INTRA-BEAM SCATTERING}

Beam growth caused by intra-beam scattering (IBS) is of primary concern during both injection and storage of the heavy ion beams in RHIC. At injection, the IBS growth time for the momentum spread is about 3 minutes. Alternate filling of the two rings, each with 60 bunches, needs to be done within about 1 minute to prevent difficulty in transition crossing and top-energy RF recapture. At storage, emittance growth occurs in both the transverse and longitudinal dimension. Collimation systems are designed to remove particles escaped from the RF buckets.

\subsection{Beam Rest Frame Hamiltonian}

Intra-beam scattering mechanism can be described using the rest frame $(x, y, z, t)$ of the circulating synchronous particle. Measure dimensions in units of the characteristic distance $\xi_{0}$ with $\xi_{0}^{3}=r_{0} \rho^{2} / \beta^{2} \gamma^{2}$, time in units of $\rho / \beta \gamma c$, and energy in units of $\beta^{2} \gamma^{2} Z^{2} e^{2} / 4 \pi \epsilon_{0} \xi_{0}$, where $r_{0}=Z^{2} e^{2} / 4 \pi \epsilon_{0} m_{0} c^{2}$ is the classical radius, $\beta c$ and 
$\gamma m_{0} c^{2}$ are the velocity and energy of the synchronous particle, and $\rho$ is the radius of curvature in bending regions of magnetic field $B_{0}$. The Hamiltonian for particles in a simple system with bending dipoles and focusing quadrupoles of strength $n_{1}=-\left(\rho / B_{0}\right)\left(\partial B_{y} / \partial x\right)$ is $[9]$

$$
H= \begin{cases}\frac{1}{2}\left(P_{x}^{2}+P_{y}^{2}+P_{z}^{2}\right)+\frac{1}{2} x^{2}-\gamma x P_{z}+V_{C} & \text { (bending section) } \\ \frac{1}{2}\left(P_{x}^{2}+P_{y}^{2}+P_{z}^{2}\right)-\frac{n_{1}}{2}\left(x^{2}-y^{2}\right)+V_{C}+U_{s} & \text { (straight section) }\end{cases}
$$

where $U_{z}$ is the potential provided by the RF system. The Coulomb potential is non-relativistic in the rest frame:

$$
V_{C}=\sum_{j} \frac{1}{\sqrt{\left(x_{j}-x\right)^{2}+\left(y_{j}-y\right)^{2}+\left(z_{j}-z\right)^{2}}} .
$$

In terms of dispersion function $D$ and betatron displacements $\beta_{x, y}$, this Hamiltonian is transformed to

$$
\tilde{H}=\frac{1}{2}\left(P_{\beta_{x}}^{2}+P_{\beta_{y}}^{2}\right)+\frac{1-\gamma^{2} F_{z}}{2} P_{z}^{2}+V_{C}+U_{z},
$$

where

$$
F_{z}=\left\{\begin{aligned}
D+D D^{\prime \prime}+\left(D^{\prime}\right)^{2} & \text { (bending section) } \\
D D^{\prime \prime}+\left(D^{\prime}\right)^{2} & \text { (straight section) }
\end{aligned}\right.
$$

and

$$
\left\langle F_{z}\right\rangle=\frac{1}{\gamma_{T}^{2}} .
$$

Below transition energy, $\gamma<\gamma_{T}$, particles are in a positive-mass regime. In an idealized case that the machine lattice is uniform along the ring circumference, the Hamiltonian in the rest frame is time-independent. The particle system is thus conscrved, so does the total temperature of the beam in the rest frame. The heat can be transfered from the high temperature to the low temperature direction. The system eventually reaches an equilibrium state when the temperature (i.e. rest-frame velocity) is the same in all directions.

In an actual alternating-gradient focusing ring, the beam sees a time dependent potential modulated by the ring lattice frequency. The beam structure absorbs "phonons" and heats up [10]. Intra-beam multiple scattering manifests as a mixture of thermal equalization and temperature growth asymptotically approaching equal temperature in all directions in the rest frame.

Above transition energy, $\gamma>\gamma_{T}$, the beam is in a negative-mass regime. The Hamiltonian (Eq. 3) indicates that even in the case of a uniform machine lattice, beam temperature can grow simultaneously in the longitudinal and transverse directions. 


\subsection{IBS Growth Scaling Laws}

Energy exchange and temperature increase in the beam rest frame manifest as variation of beam emittance and momentum spread in the laboratory frame. In the laboratory frame, the rate of emittance and momentum growth is usually obtained $[11,12]$ assuming multiple small-angle scattering among Gaussian-distributed beams. In the case that $D / \beta_{x}^{1 / 2}$ is nearly constant (e.g. for FODO lattice), the growth rate formula can be simplified into the following expression $[13,3]$,

$$
\left[\begin{array}{l}
\frac{1}{\sigma_{p}} \frac{d \sigma_{p}}{d t} \\
\frac{1}{\sigma_{x}} \frac{d \sigma_{x}}{d t} \\
\frac{1}{\sigma_{y}} \frac{d \sigma_{y}}{d t}
\end{array}\right]=\frac{Z^{4} N}{A^{2}} \frac{r_{0}^{2} m_{0} c^{2} L_{c}}{8 \gamma \epsilon_{x} \epsilon_{y} S_{r m s}} F(\chi)\left[\begin{array}{l}
n_{b}\left(1-d^{2}\right) \\
-a^{2} / 2+d^{2} \\
-b^{2} / 2
\end{array}\right]
$$

where $L_{C} \approx 20$ is the Coulomb logarithm, $\epsilon_{x, y}=\beta \gamma \sigma_{x, y}^{2} / \beta_{x, y}=\epsilon_{N} / 6$ is the normalized rms transverse emittance, $S_{r m s}=\pi m_{0} c^{2} \beta \gamma \sigma_{s} \sigma_{p} / c A=S / 6$ is the rms longitudinal bunch area in phase space, $\chi=\left(a^{2}+b^{2}\right) / 2, d=\frac{D \sigma_{p}}{\left(\sigma_{x}^{2}+D^{2} \sigma_{p}^{2}\right)^{1 / 2}}, a=\frac{\beta_{x} d}{D \gamma}$, $b=\frac{\beta_{y} \sigma_{x}}{\beta_{x} \sigma_{y}} a, n_{b}$ is equal to 1 if the beam is azimuthally bunched, and is equal to 2 if it is not. For azimuthally bunched beams, $\sigma_{s}$ is the rms bunch length and $N$ is the number of particles per bunch; for unbunched beams, $N$ is the total number of particles and $\sigma_{s}=\sqrt{\pi} R$. In Eq. $6, F(\chi)$ is an analytic function given by

$$
F(\chi)=\frac{-3+(1+2 \chi) I(\chi)}{1-\chi}
$$

where

$$
I(\chi)= \begin{cases}\frac{1}{\sqrt{\chi(\chi-1)}} \operatorname{Arth} \sqrt{\frac{\chi-1}{\chi}} \quad & x \geq 1 \\ \frac{1}{\sqrt{\chi(1-\chi)}} \arctan \sqrt{\frac{1-\chi}{\chi}} & x<1\end{cases}
$$

The growth rates are linearly proportional to the number of the particle $N$ in the beam, and are strongly dependent $\left(\sim Z^{4} / A^{2}\right)$ on the charge state of the particle. Except for the form factors $\chi, d, a$, and $b$ that depend on the ratio of the beam amplitudes in different dimension, the rates are inversely proportional to the six dimensional phase space area. Below transition energy, the asymptotic distribution corresponds to the condition

$$
\left\langle\frac{\sigma_{x}}{\beta_{x}}\right\rangle \approx\left\langle\frac{\sigma_{y}}{\beta_{y}}\right\rangle \approx \frac{\sigma_{p}}{\gamma}, \quad \gamma \ll \gamma_{T}
$$


Above transition energy, the asymptotic distribution corresponds to the condition

$$
\sqrt{n_{b} n_{c}}\left\langle\sigma_{x}\right\rangle \approx\langle D\rangle \sigma_{p}, \quad \gamma \gg \gamma_{T}
$$

where $n_{c}$ is equal to 1 if the horizontal and vertical motion are uncoupled, and is equal to 2 if they are fully coupled. In order to confine the horizontal emittance growth, we intensionally couple the horizontal and vertical motion. The growth rates at high energy become

$$
\left[\begin{array}{c}
\frac{1}{\sigma_{p}} \frac{d \sigma_{p}}{d t} \\
\frac{1}{\sigma_{x}} \frac{d \sigma_{x}}{d t}
\end{array}\right]=\frac{Z^{4} N}{A^{2}} \frac{\pi r_{0}^{2} m_{0} c^{2} L_{c}}{16 \gamma_{T} \epsilon_{x} \epsilon_{y} S_{r m s}}\left[\begin{array}{l}
n_{b}\left(1-d^{2}\right) / d \\
d / n_{c} .
\end{array}\right], \quad\left(\gamma \gg \gamma_{T}\right)
$$

which is to the first order independent of the beam energy.

\subsection{Fokker-Planck Approach and IBS Beam Loss}

In order to evaluate the beam intensity lifetime, we use the Fokker-Planck equation to describe the evolution of particle distribution in the phase space. The general 6 dimensional ( $6-\mathrm{D}$ ) equation can be greatly simplified by the fact that the IBS growth time is typically much longer than the synchrotron-oscillation period, which is again much longer than the multiple collision relaxation time. Since the leading source of beam loss is expected to be in the longitudinal direction due to the limited voltage of the RF system, we further assume in the transverse directions a time-evolving Gaussian distribution. After averaging over the machine circumference and the synchrotron phase for all the particles involved in the collision [14], we obtain a 1-D Fokker-Planck equation of the density function $\Psi(J)$ in the longitudinal direction in terms of the action variable $J$,

$$
\frac{\partial \Psi}{\partial t}=-\frac{\partial}{\partial J}(F \Psi)+\frac{1}{2} \frac{\partial}{\partial J}\left(D \frac{\partial \Psi}{\partial J}\right), \text { with } \begin{cases}J=0: & -F \Psi+\frac{D}{2} \frac{\partial \Psi}{\partial J}=0 \\ J=J_{\max }: & \Psi=0 .\end{cases}
$$

Here, the drift cuefficient is given by the expression

$$
F(J)=\left.\left.\oint \frac{2 d s}{\pi R} \int_{0}^{\frac{1}{4}} d Q \frac{\partial W}{\partial J}\right|_{\phi} ^{-1}(Q, J) \int_{J_{m เ n}}^{\hat{J}} \frac{\partial W}{\partial J}\right|_{\phi}\left(Q^{\prime}, J^{\prime}\right)\left[A_{F}\left(\lambda_{1}\right)+A_{F}\left(\lambda_{2}\right)\right] \Psi\left(J^{\prime}\right) d J^{\prime}
$$

and the diffusion coefficient is given by the expression 
$D(J)=\left.\oint \frac{2 d s}{\pi R} \int_{0}^{\frac{1}{4}} d Q\left[\left.\frac{\partial W}{\partial J}\right|_{\phi} ^{-1}(Q, J)\right]^{2} \int_{J_{\min }}^{\hat{J}} \frac{\partial W}{\partial J}\right|_{\phi}\left(Q^{\prime}, J^{\prime}\right)\left[A_{D}\left(\lambda_{1}\right)+A_{D}\left(\lambda_{2}\right)\right] \Psi\left(J^{\prime}\right) d J^{\prime}$

where

$$
A_{F}(\lambda)=-\frac{2 Z^{4} r_{0}^{2} L_{c} E}{A^{2} \beta^{2} \gamma^{4}} \frac{I_{F}(\lambda)}{\sigma_{x} \sigma_{y}}, \quad A_{D}(\lambda)=\frac{Z^{4} r_{0}^{2} L_{c} E^{2}}{A^{2} \gamma^{3} h \omega_{s}} \frac{I_{D}(\lambda)}{\sigma_{x} \sigma_{y}}
$$

$\omega_{s}$ is the revolution frequency,

$$
\begin{gathered}
\lambda_{1,2}=\frac{h \omega_{s} g}{\gamma \beta^{2} E}\left(W \mp W^{\prime}\right), g=\frac{1}{2} \sqrt{\frac{\beta \gamma \beta_{x, y}}{\epsilon_{x, y}}}, \\
\left.\frac{\partial W}{\partial J}\right|_{\phi} ^{-1}(Q, J)=8 k K(k) \cos 2 \pi Q\left[1-4 \xi \sin ^{2} 2 \pi Q+O\left(\xi^{2}\right)\right],
\end{gathered}
$$

and $\xi=\exp \left[-\pi \mathrm{K}^{\prime}(k) / \mathrm{K}(k)\right]$ and $\mathrm{K}^{\prime}(k)=\mathrm{K}\left(\sqrt{1-k^{2}}\right)$. The first integrals in Eqs. 11 and 12 represent the average over the machine lattice; the second integral represents the average over synchrotron-oscillation phase; while the third integral describes particles of different action $J^{\prime}$ involved in the collision. The integration over $J^{\prime}$ is performed such that $k\left(J^{\prime}\right) \sin 2 \pi Q^{\prime} \approx \sin [\phi(Q, J) / 2]$, extending from $J_{\min }$ to the bunch edge $\hat{J}$, with $k\left(J_{\min }\right) \approx[\sin \phi(Q, J) / 2]$. For a round beam with near constant $D / \beta_{x}^{1 / 2}$, we have

$$
\begin{aligned}
& I_{F}(\lambda)=2 g^{2} \operatorname{sgn}(\lambda) e^{-\left(D \gamma \lambda / 2 \sigma_{x}\right)^{2}}\left\{1-\sqrt{\pi}|\lambda| e^{\lambda^{2}}[1-\Phi(\lambda)]\right\}, \\
& I_{D}(\lambda)=g e^{-\left(D \gamma \lambda / 2 \sigma_{x}\right)^{2}}\left\{\sqrt{\pi}\left(1+2 \lambda^{2}\right) e^{\lambda^{2}}[1-\Phi(\lambda)]-2|\lambda|\right\},
\end{aligned}
$$

where $\Phi$ is the error function, and $\operatorname{sgn}(\lambda)$ is 1 if $\lambda \geq 0$, and is -1 if otherwise.

Starting from an initial distribution, Eq. 10 can be iterated to yield the time evolution of the longitudinal particle distribution, as shown in Fig. 1. Based on this information, evolution of the transverse beam dimension is obtained from the growth rate formulae given in Section 2.2. Beam loss through the RF bucket boundary is evaluated from the reduction of the integrated density $\Psi(J)$ over $J$. Typically, the longitudinal distribution under intra-beam scattering is Gaussian-like with zero density at the edge of the RF bucket, as shown in Fig. 1.

\subsection{Luminosity and Beam Cooling}

Due to emittance growth and intensity loss caused by intra-beam scattering, the luminosity in RHIC is significantly reduced at the early stage of storage, as 


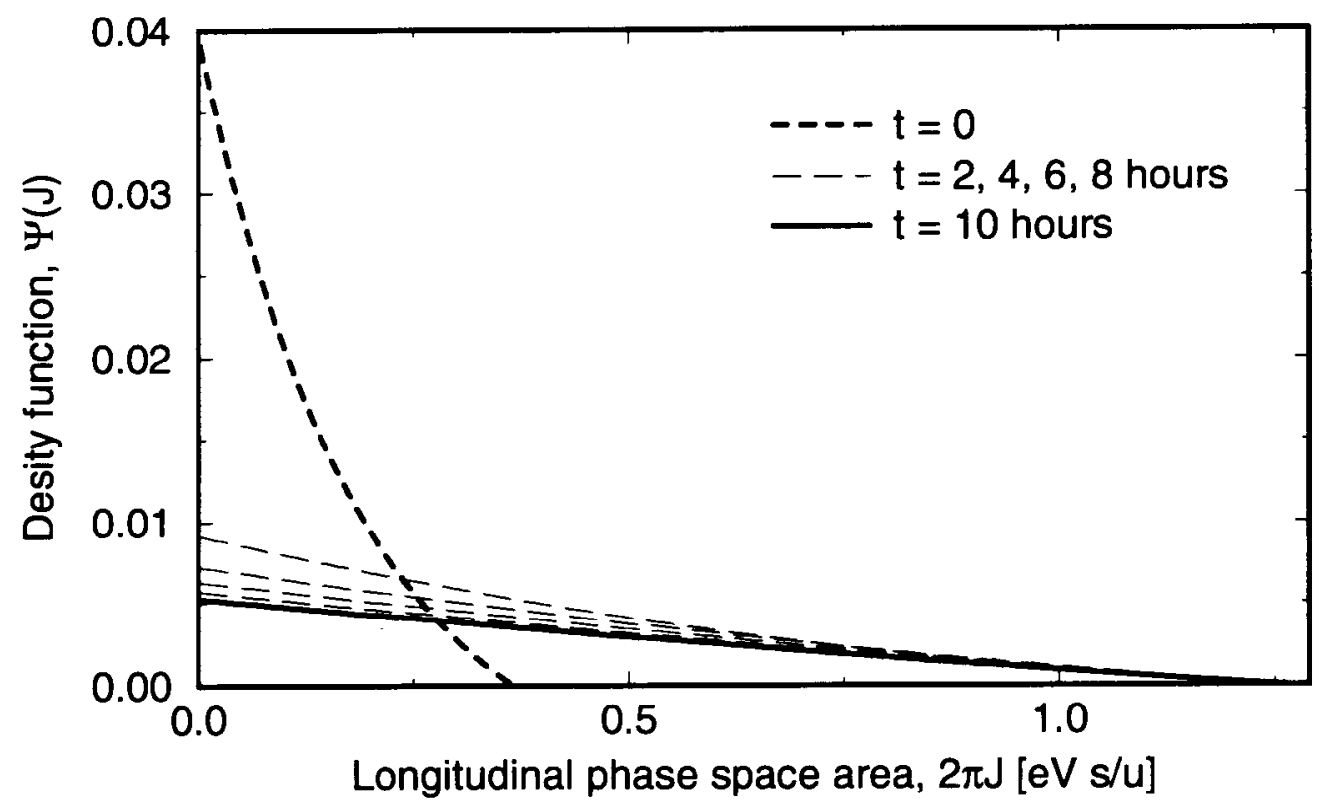

FIGURE 1. Evolution of the longitudinal density distribution under intra-beam scattering during the 10 hour storage in RHIC. The edge of RF bucket corresponds to $1.29 \mathrm{eV} \cdot \mathrm{s} / \mathrm{u}$.

shown by the solid line in Fig. 2. Increasing peak RF voltage only improves the performance modestly, since transverse growth is strong and momentum acceptance can be a problem [7]. The ultimate improvement can be achieved if beam cooling methods are adopted. Fig. 2 shows the improvement of integrated luminosity over a 10-hour store if transverse and longitudinal stochastic cooling [4] are employed. Cooling in both longitudinal and transverse planes provides an effective method to compensate for the beam growth, particle loss, and luminosity deterioration caused by IBS. With longitudinal and transverse cooling of bandwidth 4-8 $\mathrm{GHz}$, the longitudinal beam loss resulted from the inadequacy of the RF voltage can be eliminated, and the transverse normalized beam emittance can be confincd to about $30 \mathrm{~mm} \cdot \mathrm{mr}$. With this scenario, the $\beta^{*}$ at the crossing point can be lowered under 1 meter without exceeding the transverse aperture limit at the focusing triplets. The integrated luminosity can be increased by at least a factor of 2 during the 10-hour storage period. Experimentally, however, stochastic cooling studies for bunched beam at the Tevatron indicates unexpected difficulty $[15,16]$ due to large coherent signal saturation. Nevertheless, stochastic cooling in RHIC is expected to be much easier since the beam, instead of tightly bunched, occupies the entire RF bucket due to IBS. Also, the high charge state greatly improves signal-to-noise ratio. Recently, electron cooling has also been studied for RHIC employing separate electron storage rings for electron damping [17]. 


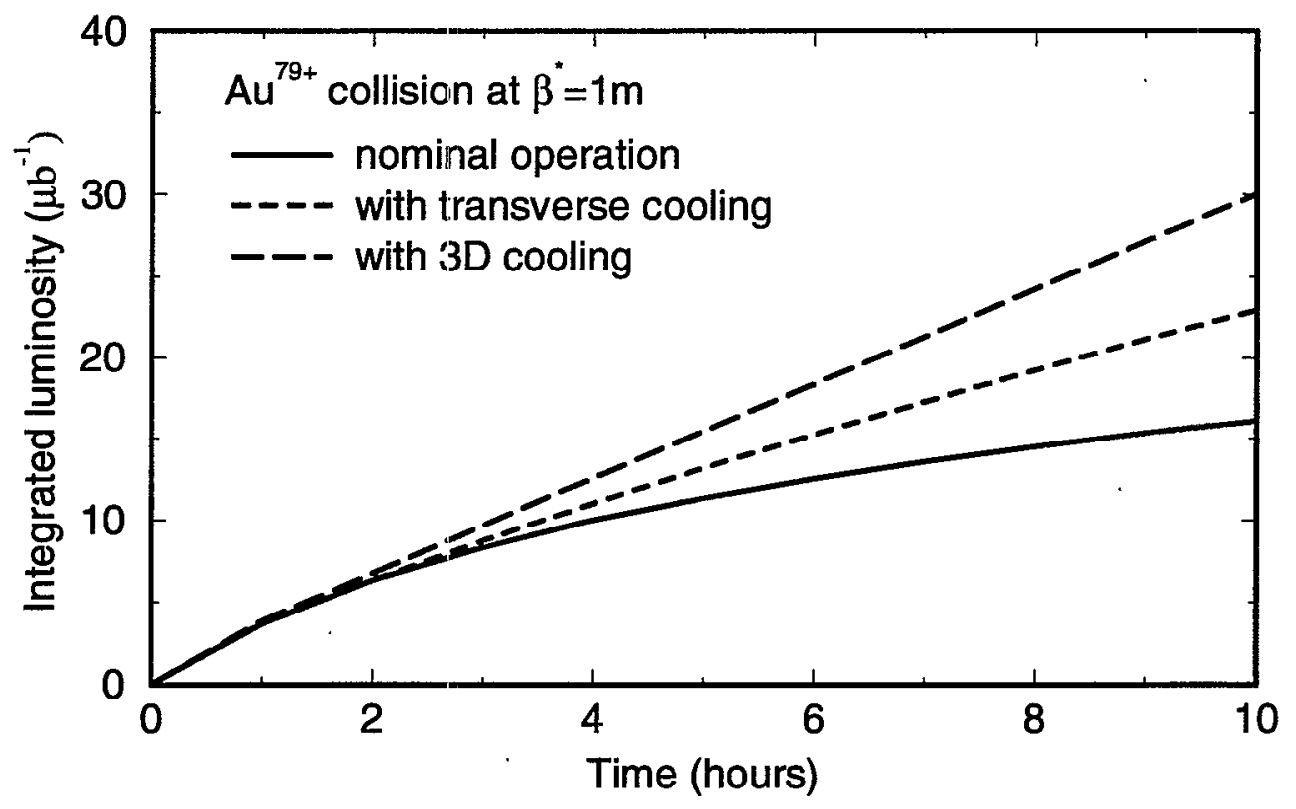

FIGURE 2. Integrated luminosities during a 10-hour store for a) the nominal operation (without cooling), b) with transverse stochastic cooling, and c) with both transverse and longitudinal stochastic cooling. The bandwidth of the cooling system is assumed to be $4-8 \mathrm{GHz}$.

\section{TRANSITION CROSSING}

Transition energy crossing is likely to be the "bottle neck" for RHIC acceleration. In the presence of the beam self field, the bunch is mismatched to the RF bucket upon the shift of synchronous phase at crossing. The mismatch increases the momentum spread, enhancing the chromatic nonlinear effect and leading to particle loss. In the absence of a transition jump, this mechanism alone accounts for an intensity loss of about $70 \%$ at transition.

\subsection{Non-Adiabatic Regime Formalism}

During acceleration, the longitudinal particle motion is non-adiabatic within a characteristic time $\pm T_{c}$ near transition energy $\gamma_{T}$

$$
T_{c}=\left(\frac{\pi E \beta^{2} \gamma_{T}^{3}}{Z e V\left|\cos \phi_{s}\right| \dot{\gamma} h \omega_{s}^{2}}\right)^{\frac{1}{3}}
$$

where $E$ is the total energy of the particle, $\dot{\gamma}$ is the ramp rate, $\phi_{s}$ is the synchronous phase, and $h$ and $V$ are harmonic number and voltage of the RF system. The 
dynamics can be best described by the longitudinal amplitude function $\beta_{L}$ given approximately by [9]

$$
\frac{\beta_{L}}{k T_{c}}=\frac{\pi}{3} x\left[\mathrm{~J}_{-\frac{1}{3}}^{2}(y)+\mathrm{N}_{-\frac{1}{3}}^{2}(y)\right] \approx 1.58-1.15 x
$$

where $y=2 x^{3 / 2} / 3, x=|\Delta t| / T_{c}, k=Z e V\left|\cos \phi_{s}\right| / 2 \pi h$, and $\Delta t$ is the time delay from $\gamma_{T}$. With a normalized time $d \tau=k d t$, the longitudinal motion is described by a Hamiltonian expressed in terms of the action-angle variables $\varphi$ and $J$ as

$$
H(\varphi, J ; \tau)= \pm J / \beta_{L}
$$

The synchrotron frequency is

$$
\Omega_{s}=k \beta_{L}^{-1}
$$

approaching a minimum but non zero value of $\Omega_{s}(0)=0.63 T_{c}^{-1}$ at transition. The maximum excursions in the RF phase $\phi$ and energy deviation $W=\Delta E / h \omega_{s}$ are

$$
\hat{\phi}=\sqrt{2 \gamma_{L} J}, \text { and } \hat{W}=\sqrt{2 \beta_{L} J}
$$

where $1+\alpha_{L}^{2}=\beta_{L} \gamma_{L}$. For a bunch of phase space area $S=2 \pi J$ at transition, the $\mathrm{RF}$ phase spread of the bunch reaches the minimum value

$$
\hat{\phi}=0.52\left(S / k T_{c}\right)^{1 / 2}
$$

while the momentum spread reaches the maximum value

$$
(\Delta \hat{p} / p)=0.71 h \omega_{s}\left(k T_{c} S\right)^{1 / 2} / E \beta^{2} .
$$

\subsection{Chromatic Nonlinearity and Self-Field Mismatch}

The chromatic nonlinear effect $[18,9]$ originates from the intrinsic mistiming among particles of different momentum deviation crossing transition, as shown in Fig. 3. Using the perturbation formalism for the non-adiabatic regime $[19,20,5]$, the Hamiltonian system including the nonlinearity can be solved to obtain the longitudinal emittance growth during transition

$$
\frac{\Delta S}{S} \approx \begin{cases}0.76 \frac{T_{n l}}{T_{c}}, & \text { for } T_{n l} \ll T_{c} \\ e^{\frac{4}{3}\left(\frac{T_{n l}}{T_{c}}\right)^{3 / 2}}-1, & \text { for } T_{n l} \geq T_{c}\end{cases}
$$

where the total nonlinear time $\pm T_{n l}$ is given by 


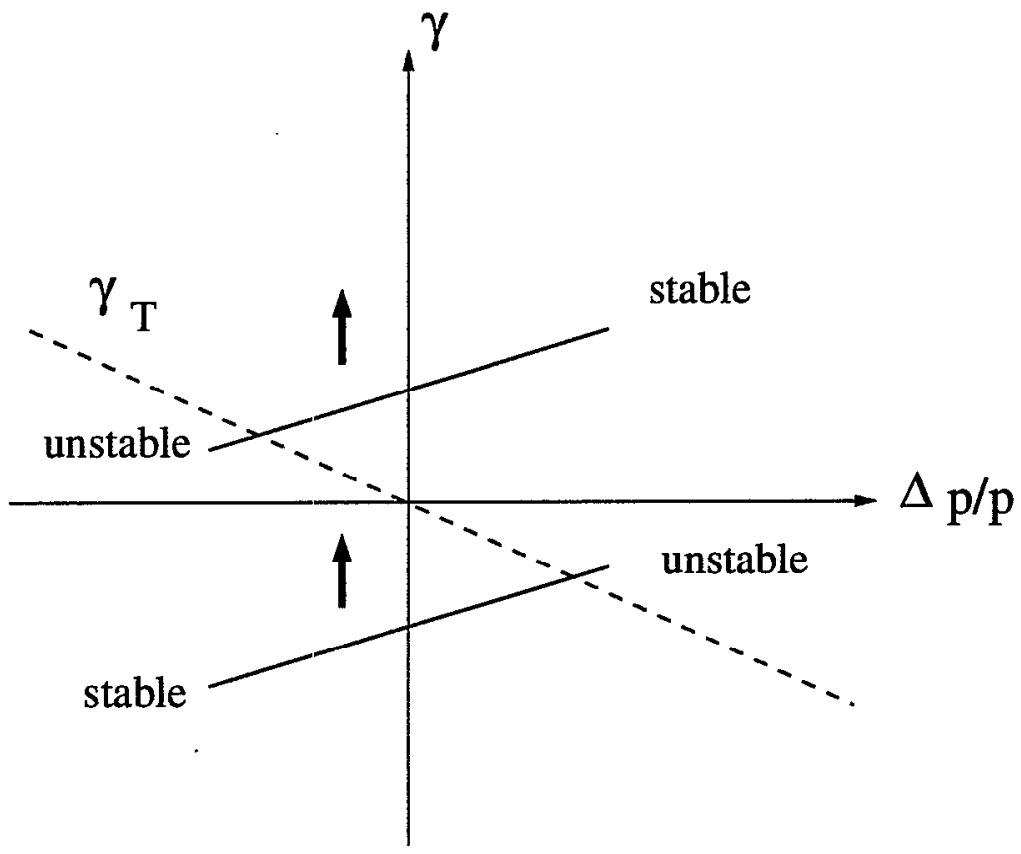

FIGURE 3. Mechanism of chromatic nonlinear effect at transition crossing. Part of the beam is unstable below transition before the RF phase switch, while the other part is unstable above transition.

$$
T_{n l}=\left|\left(\alpha_{1}+\frac{3 \beta^{2}}{2}\right)\right| \frac{\sqrt{6} \hat{\sigma}_{\Delta p / p} \gamma_{T}}{\dot{\gamma}}
$$

where $\alpha_{1}$ is the nonlinear momentum compaction factor [9]. In the absence of a transition jump, the chromatic effect alone (low intensity case) accounts for about $20 \%$ beam loss at transition in RHIC.

Both reactive and resistive impedances cause mismatch in the nominal bunch shape at the time the synchronous phase is jumped at transition. A reactive impedance changes the focusing force of the RF system differently below and above transition. The amount of mismatch is then proportional to the ratio of the self field to the RF field provided by the accelerating cavities. Again, we use the perturbation approach $[21,5]$ to solve the Hamiltonian system in the non-adiabatic regime that includes the self field potential. For a parabolic distribution, the effective increase in the bunch area due to the mismatch, induced by a coupling impedance $\left|Z_{L} / n\right|$ around the bunch frequency, is

$$
\frac{\Delta S}{S}=\frac{2 h \hat{I}\left|Z_{L} / n\right|}{\hat{V}\left|\cos \phi_{s}\right| \hat{\phi}^{2}}
$$

where

$$
\hat{I}=\frac{3 h N Z e \omega_{s}}{4 \hat{\phi}}
$$


is the peak current. The effect is usually a quadrupole-mode bunch tumbling and growth. For RHIC, the space charge impedance is about $\left|Z_{L} / n\right| \approx 1.2 \Omega$. The induced force stretches the bunch momentum around transition. This momentum increase enhances the chromatic nonlinear effect, together resulting in a beam loss of about $70 \%$ in the absence of a transition jump.

\subsection{Microwave Instability at Transition}

As synchrotron frequency approaches a minimum value (Eq. 20) at transition, microwave instability is likely to occur. For a bunched beam, the instability threshold is inversely proportional to the ratio between the self field and the focusing field, i.e. inversely proportional to the bunch length (Eq. 21) to the cubic power. Quantitatively, we can solve the Vlasov equation in terms of the action variable using the non-adiabatic regime formalism [21]. An exact solution for the instability threshold can be obtained for the parabolic beam distribution as

$$
D_{\|} \approx \frac{4 h \hat{I}\left|Z_{\|} / n\right|}{9 V\left|\cos \phi_{s}\right| \hat{\sigma}_{\phi}^{2}} \geq 1 .
$$

A capacitive (or inductive) longitudinal coupling impedance $Z_{\|}$at a broad-band frequency will cause a microwave instability during a time

$$
T_{m w} \approx 1.37\left(D_{\|}-1\right) T_{c}
$$

after (or before) transition. In the absence of the transition jump in RHIC, the beam at the nominal intensity is near the instability threshold due to the space charge force alone.

\subsection{Compensation with a Transition Jump}

The cause of strong chromatic nonlinear effect and self-field effect at RHIC is the slow ramp rate of the superconducting magnets. The most efficient way to reduce these effects is to effectively increase the crossing rate by a transition energy jump, which is achieved by pulsing two families of quadrupole correction magnets for about $60 \mathrm{~ms}$. The change of $\gamma_{T}$ of the lattice is about \pm 0.4 units, effectively increasing the crossing rate by about a factor of 10 .

A key issue in the design of transition jump schemes is to minimize the enhancement of machine lattice distortion. As experimentally demonstrated on the AGS $[22,23]$ (Fig. 4), the jump could significantly enhance the nonlinear factor $\alpha_{1}$, and sextupoles were arranged to reduce the nonlinearity. With RHIC, we designed a "matched, first order" transition jump scheme [6] with which the longitudinal emittance growth can be limited to less than $20 \%$ at transition with minimum disruption to the transverse particle motion. Fig. 5 shows the residual beam growth at transition in the presence of the design transition jump. 


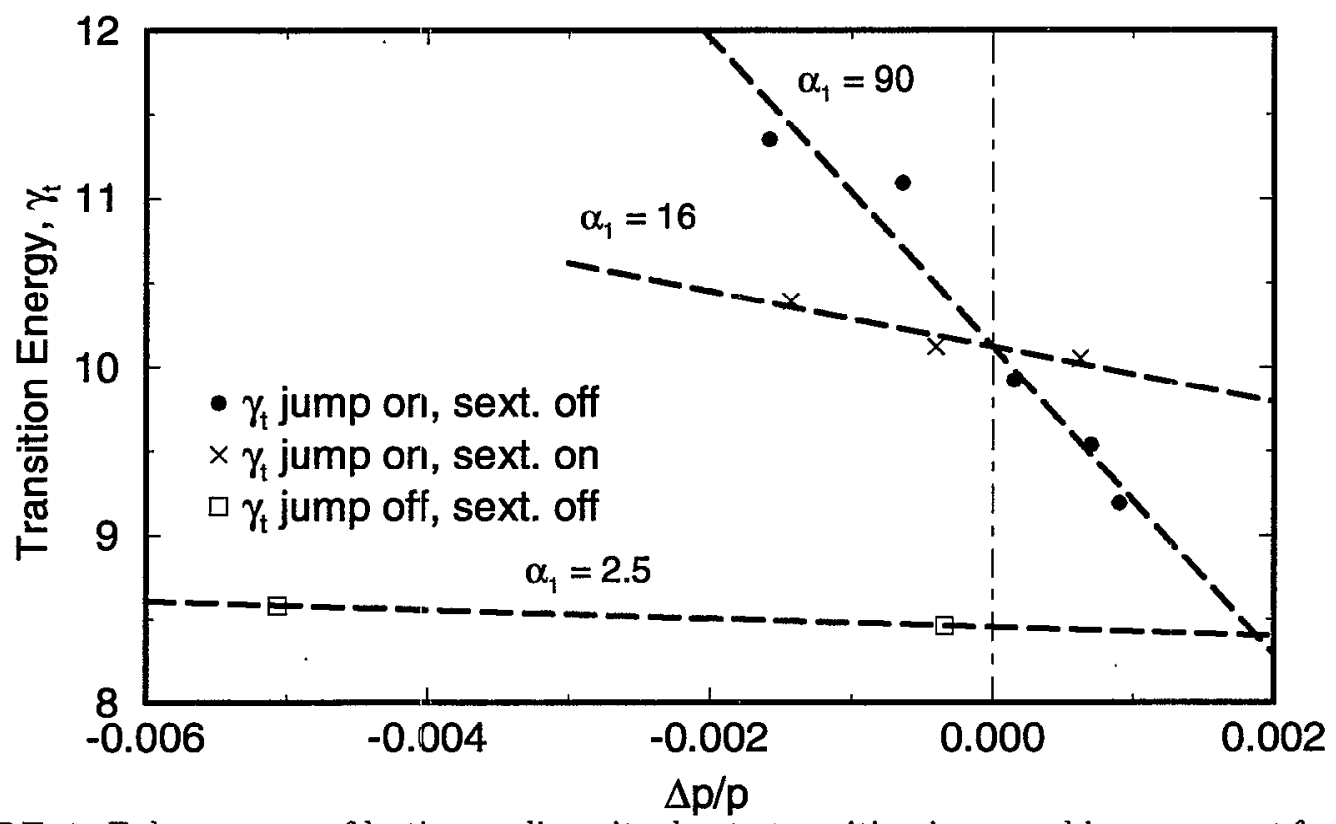

FIGURE 4. Enhancement of lattice nonlinearity due to transition jump and improvement from chromaticity sextupoles. The experimental study was done at the AGS with gold beam crossing transition. A "second order" transition jump scheme was used.

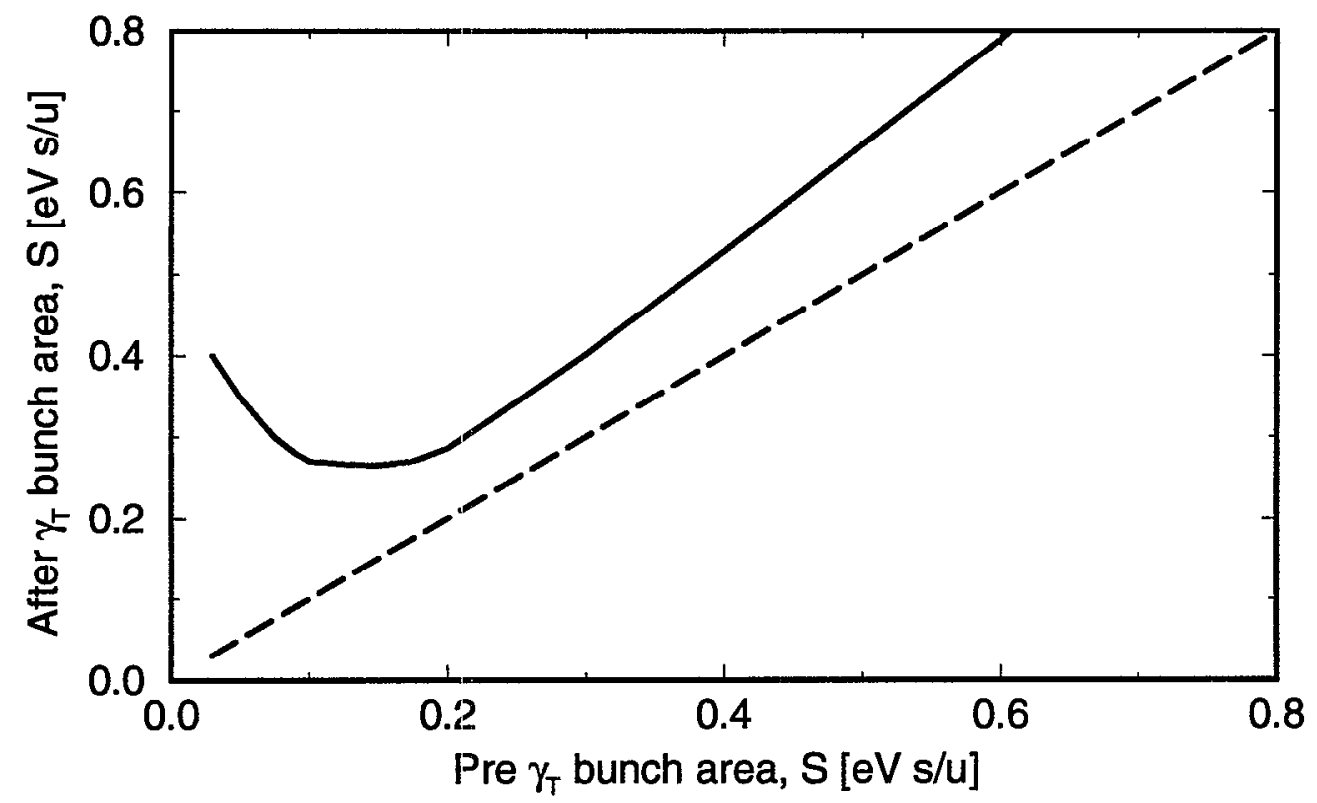

FIGURE 5. Tongitudinal beam growth at RHIC transition energy. The growth for beams with smaller bunch area is mainly due to self field mismatch and microwave instability, while the growth for beams with larger bunch area is mainly due to thc nonlincarity in momentum. The design bunch area is $0.3 \mathrm{eV} \cdot \mathrm{s} / \mathrm{u}$. 


\section{SPACE CHARGE AND BEAM-BEAM EFFECTS}

The mean field of the beam space charge modulates the transverse tunes of the particles and produces a tune spread that may compromise the dynamic aperture of the machine. The tune spread $\left|\Delta Q_{s c}\right|$ is the largest at the injection energy,

$$
\left|\Delta Q_{s c}\right|=\frac{3 Z^{2} N r_{0}}{2 \pi A \beta \gamma^{2} \epsilon_{N} B_{f}} \approx \begin{cases}0.030 \frac{N}{10^{9}} \frac{10[\mu \mathrm{m}]}{\epsilon_{N}} & \text { for gold } \\ 0.015 \frac{N}{10^{11}} \frac{20[\mu \mathrm{m}]}{\epsilon_{N}} & \text { for proton }\end{cases}
$$

where $\epsilon_{N}$ is the normalized $95 \%$ emittance, and

$$
B_{f}=\frac{\sigma_{s}}{\sqrt{2 \pi} R}
$$

is the bunching factor.

The mean field of the space charge between the colliding beams produces a tune spread at collision. With zero crossing angle, the head-on beam-beam tune spread per crossing is [24]

$$
\left|\Delta Q_{b b}\right|=\frac{3 Z^{2} N r_{0}}{2 A \epsilon_{N}} \approx \begin{cases}0.0012 \frac{N}{10^{9}} \frac{20[\mu \mathrm{m}]}{\epsilon_{N}} & \text { for gold } \\ 0.0037 \frac{N}{10^{11}} \frac{20[\mu \mathrm{m}]}{\epsilon_{N}} & \text { for proton }\end{cases}
$$

which are independent of the energy and $\beta$ functions. Since the beams are separated immediately around the interaction point before entering into the triplet region. there are no parasitic collisions for the nominal storage scenario of 60 bunches per ring.

\section{IMPEDANCES AND INSTABILITIES}

In RHIC, the narrow-band impedance spectrum is dominated by RF cavities, while the broadband impedance spectrum is dominated by the resistive wall effect at very low frequencies and by kicker magnets in the intermediate frequency range up to $3 \mathrm{GHz}[8]$.

The threshold for longitudinal microwave instabilities is lowest for heavy ions during transition crossing, and for protons during re-bucketing [8]. Transverse and longitudinal dampers are planned to compensate for coupled bunch instabilities [8]. The chromaticities of the machine are designed to shift from negative to positive for about 5 units in $30 \mathrm{~ms}$ at transition to prevent potential head-tail instability. Electron cloud effect has been [25] found to be tolerable during nominal operation with 60 bunches per ring. 


\section{CONCLUSIONS}

Intra-beam scattering is the leading mechanism of luminosity degradation, emittance growth, and beam loss for RHIC. The beam is intentionally coupled in the transverse directions to reduce horizontal beam growth. The effects can be compensated by increasing the RF voltage, by a quicker re-filling, and ultimately by beam cooling methods. Transition crossing in RHIC is complicated by chromatic nonlinear effect, beam self-field mismatch, and microwave instability. The effects will be compensated by a first-order transition jump.

In year 2000, RHIC will enter its first year of operation. The machine will provide a test bed to verify the predictions discussed in this paper.

\section{REFERENCES}

1. RHIC Design Manual, Brookhaven National Laboratory, Upton, New York (1998).

2. J. Wei, M. Harrison, The RHIC Project - Design, Status, Challenges, and Perspectives, XVI RCNP Osaka Inter. Sym. on Multi-GeV High-Performance Accelerators and Related Technology (Osaka, 1997).

3. J. Wei, Evolution of Hadron Beams under Intra-beam Scattering, Partical Accelerator Conference (Washington, D.C., 1993), p.3653.

4. J. Wei, Stochastic Cooling and Intra-Beam Scattering in RHIC, Workshop on Beam Cooling and Related Topics (Montreux, 1994), p. 132 (CERN 94-03).

5. J. Wei, Longitudinal Dynamics of the Non-Adiabatic Regime on Alternating-Gradient Synchrotrons, Ph. D. dissertation, Stony Brook, New York (1990); revised 1994.

6. S. Peggs, S. Tepikian, D. Trbojevic, A First Order Transition Jump at RHIC, Particle Accelerator Conference (Washington, D.C., 1993), p. 168.

7. J. Wei, J. Kewisch, V. Ptitsin, J. Rose, RHIC Longitudinal Parameter Revision, European Particle Accelerator Conference (Stockholm, 1998) p. 377.

8. M. Blaskiewicz, D.P. Deng, W.W. MacKay, V. Mane, S. Peggs, A. Ratti, J. Rose, T. Shea, J. Wei, Collective instabilities in RHIC, Rev. 1.3, RHIC/AP/36, Brookhaven National Laboratory (1994).

9. Handbook of Accelerator Physics and Engineering, edited by A. Chao and M. Tigner, World Scientific, Singapore, 1999.

10. X-P. Li, A.M. Sessler, J.Wei, Crystalline Beam in a Storage Ring: How Long Can It Last? European Accelerator Conference (London, 1994), p. 1379.

11. A. Piwinsky, Intra-Beam-Scattering, CERN 92-01, CERN Accelerator School (Gifsur-Yvette, Paris, 1984), p. 405.

12. J. Bjoken, S.Mtingwa, Intrabeam Scattering, Particle Accelerators, 13 (1983) 115.

13. G. Parzen, Intrabeam Scaltering at High Energies, Nuclear Instruments \& Methods, A256 (1987) 231.

14. J.Wei and A.G.Ruggiero, Beam Life-Time with Intra-Beam Scattering and Stochastic Cooling, Particle Accelerator Conference (San Francisco, 1991), p. 1869.

15. J. Marriner, Theory, Technology, and Technique of Stochastic Cooling, Workshop on Beam Cooling and Related Topics (Montreux, 1994), p. 14 (CERN 94-03). 
16. D. Möhl, The Status of Stochastic Cooling, Nuclear Instruments \& Methods, A 391 (1997) 164.

17. A. Burov, V. Danilov, P. Colestock, Ya. Derbenev, Electron Cooling for RHIC, Fermilab-TM-2058 (1998).

18. K. Johnsen, Effects of Non-Linearities on the Phase Transition, Proceedings of CERN Symposium on High-Energy Accelerators and Pion Physics (Geneva, 1956), p. 106.

19. K. Takayama, Phase Dynamics near Transition Energy in the Fermilab Main Ring, Particle Accelerators, 14 (1984) 201.

20. S.Y. Lee and J. Wei, Nonlinear Synchrotron Motion Near Transition Energy in RHIC, European Particle Accelerator Conference (Rome, 1988), p.764.

21. J. Wei, S.Y.Lee, Microwave Instability near Transition Energy, Particle Accelerators, 28 (1990) 77.

22. J. Wei, A. Warner, L. Ahrens, J.M. Brennan, W.W. Mackay, S. Peggs, A. Ratti, K. Reece, T. Roser, W.A. Ryan, C. Saltmarsh, T. Satogata, D. Trbojevic, W. Van Asselt, Experimental Study of Slow-Rate Transition Crossing in AGS, European Accelerator Conference (London, 1994), p. 976.

23. J. Wei, J.M. Brennan, L. Ahrens, M. Blaskiewicz, D-P. Deng, W.W. Mackay, S. Peggs, T. Satogata, D. Trbojevic, A. Warner, and W. Van Asselt, Effects of Enhanced Chromatic Nonlinearity during the AGS $\gamma_{T}$ Jump, Proc. 1995 Particle Accelerator Conference and International Conference on High-Energy Accelerators (Dallas, 1995), p. 3334.

24. S. Peggs, Beam-beam collisions and crossing angles in RHIC, RHIC/AP/169, Brookhaven National Laboratory (1999).

25. K.A. Drees, Beam Induced Electron Clouds at RHIC, RHIC/AP/150, Brookhaven National Laboratory (1998). 